\title{
PATTERN ADAPTATION IN CAT VISUAL CORTEX IS A CO-OPERATIVE PHENOMENON
}

\begin{abstract}
T. R. VIDYASAGAR*
Department of Neurobiology, Max-Planck Institute for Biophysical Chemistry, Goettingen, F.R.G.

Abstract--The effects of microiontophoretic application of glutamate, GABA and the GABA antagonist. bicuculline methiodide were tested on the degree of adaptation exhibited by striate cortical cells to moving sin wave grating patterns. Application of GABA, which prevents firing of the cell and thereby any fatigue of the cell, did not reduce the degree of adaptation. Administration of either glutamate or GABA, without simultaneous exposure to the adapting high-contrast gratings did not reduce the sensitivity of the cell to subsequent exposure of a low-contrast grating, showing that adaptation is not caused by the excitatory or inhibitory activity of the cell itself. Application of the GABA antagonist, bicuculline did not prevent pattern adaptation, indicating that the lowered sensitivity of the cell is not mediated by a GABAergic inhibition acting on the cell. Thus adaptation of a striate neuron is not due to altered sensitivity of the coll to a constant input but depends upon changes in the input itself. It is most likely that these changes occur in a co-operative cortical network, whose effect on individual cortical cells is mediated by intracortical excitatory connections.
\end{abstract}

After prolonged viewing of a high-contrast grating pattern, the threshold for detection of the grating is elevated, the effect being highest for the spatial frequency and orientation of the adapting grating., ${ }^{3,5}$ A possible neural correlate of this perceptual phenomenon can be seen in the responses of striate cortical cells. ${ }^{19.15 .16 .17 .19 .20 .21}$ After exposure to drifting high-contrast gratings, striate neurons show a significant reduction in sensitivity, which is especially evident when testing with low-contrast gratings. So far, studies investigating the neural basis of adaptation have mainly considered two possibilities, namely that adaptation reflects fatigue of individual neurons or that it is an after-effect of prolonged inhibition on individual cortical cells. ${ }^{4.8}$ However, it is also possible that adaptation reflects a change in the input to the cell rather than a change in the sensitivity of the cell to a fixed input. Since adaptation is not shown by neurons of the dorsal lateral geniculate nucleus (dLGN), ${ }^{17.2021}$ there is the possibility that adaptation is a cortical phenomenon occurring in a network of cells sharing similar properties like orientation and spatial frequency selectivities. In such a case. the contribution of an individual neuron to the overall adaptation may be very small, even though the effect of adaptation could be observed in the responses of all the cells in the network. Such an effect could be either due to an inhibitory influence of the network on the individual neurons or to an overall reduction of activity in a network of neurons that mutually excite each other.

*Present address: Division of Neuroscience, John Curtin School of Medical Research, Australian National University, Canberra, ACT 2601, Australia.

Abbreviations: dLGN, dorsal lateral geniculate nucleus: EPSP, excitatory postsynaptic potential.
We have tested these possibilitics by observing the effects of iontophoretic application of glutamate. GABA and bicuculline on the adaptation shown by individual striate cortical cells. Suppression of the cell's response by the administration of GABA would prevent adaptation of the cell, if fatigue due to repeated firing were the basis of adaptation. Application of glutamate alone without the presentation of the adapting grating should also cause the cell to adapt. On the other hand, if adaptation were to be an after-effect of continued inhibition acting on the cell, 4.8 administration of GABA without the presentation of the adapting pattern would reduce the sensitivity of the neuron to subsequent presentation of a pattern. If adaptation indeed depends upon changes in the input to a cell, local administration of the GABA antagonist, bicuculline would distinguish whether this effect is mediated through excitatory or inhibitory inputs to the cell.

\section{EXPERIMENTAL PROCEDURES}

\section{General}

For the initial surgery, the cats (common domestic cats, bred at the animal house of the institute) were anaesthetized with $25 \mathrm{mg} / \mathrm{kg}$ of ketamine (Parke-Davis). The surgery consisted of venous and tracheal canulations, bilateral cervical sympathectomy and a craniotomy. The animals were then maintained on a $(70: 30)$ nitrous oxide: oxygen mixture and $1 \mathrm{mg} / \mathrm{kg}$ per h of pentobarbital (Nembutal; CEVA). The adequacy of anaesthesia was assessed by monitoring the heart rate and the electroencephalogram. At the levels of anaesthesia used during the experiment, heart rate remained stable and the electroencephalogram showed slow wave activity. Muscular paralysis was achieved by the administration of $5 \mathrm{mg} / \mathrm{kg}$ per $\mathrm{h}$ of gallamine triethiodide (Sigma) and $0.1 \mathrm{mg} / \mathrm{kg}$ per $\mathrm{h}$ of pancuronium (Organon Tecknika). Artificial ventilation was adjusted to keep the end-tidal $\mathrm{CO}_{2}$ near $4 \%$. Atropine and phenylephrine (Neosynephrine) were topically applied on the eyes and $3 \mathrm{~mm}$ diameter 
artificial pupils were used. Refraction was corrected by the use of appropriate hard contact lenses that brought to focus stimuli presented on screen placed $57 \mathrm{~cm}$ from the animal's eyes. Subscapular temperature was maintained at $38 \mathrm{C}$ with a heating pad.

Penetrations were made in area 17 along the medial bank of the postlateral gyrus, usually between IIorsley-Clarke coordinates $\mathrm{Pl}$ and $\mathrm{P} 6$. The locations of cells in area 17 were also verified by histological reconstructions of the tracks from identification in $52-\mu \mathrm{m} \mathrm{Niss}$-stained sections of the electrolytic lesions made at specific recording sites. No reference will be made to the laminar location of the cells in this paper, since the number of neurons per lamina was not large enough to make reliable categorizations.

\section{Administration of drugs}

The electrode assembly used for these experiments consisted of a tungsten-in-glass recording electrode to which a number of micropipettes were glued under microscopic control. The pipettes were each filled with one of the following solutions: bicuculline methiodide (Pierce) $0.01 \mathrm{M}$ in $165 \mathrm{mM} \mathrm{NaCl}, \mathrm{pH} \mathrm{3;} \mathrm{L-glutamic} \mathrm{acid} \mathrm{(Sigma)} \mathrm{0.5} \mathrm{M,}$ $\mathrm{pH} 8$; GABA (Sigma) $0.5 \mathrm{M}, \mathrm{pH} 3$; NaCl $3 \mathrm{M}$. The effectiveness of bicuculline was always assessed by its ability to nullify the inhibitory effects of iontophoretically applied GABA on the responses of the neuron. The current of GABA for such controls was usually three to four times higher than that necessary to completely suppress the cell's response. Only those cells where an effective block of GABAergic inhibition could be established were used for further experiments with bicuculline. For more details of the iontophoretic technique and other controls, see elsewhere. ${ }^{23}$

\section{Visual stimulation}

Sin wave gratings, bars and edges could be produced on a cathode ray scope (Joyce Electronics Ltd, U.K.) using a microprocessor (CMS Cambridge, U.K.). The screen subtended $31^{\circ} \times 22^{\circ}$ of visual angle and had a mean luminance of $200 \mathrm{~cd} / \mathrm{m}^{2}$. A PDP- 11 computer controlled the presentation of grating stimuli varying in spatial frequency, temporal frequency and orientation in an interleaved tashion. After plotting orientation, spatial frequency and temporal frequency tuning curves, near optimal values were chosen for the experiments on each cell.

\section{RESULTS}

Of the neurons that were tested for adaptation in area 17 , most (15 simple and 15 complex) showed significant adaptation, reaching the criterion that adaptation to the high-contrast grating reduced the response to the test grating by at least half. Contrasts for adapting and test gratings were then so chosen that adaptation reduced the response to the test grating by $40-50 \%$. For simple cells, the amplitude of the first harmonic component was taken as the measure of response. Since complex cells do not show a modulated response to moving gratings like the simple cells, the increase in mean discharge rate above the spontaneous activity was taken as their response measure.

The adaptation to a high-contrast grating exhibited by a complex cell (A) and also a simple cell (B) is shown in Fig. 1. This adaptation occurred to about the same extent if the cell's firing during adaptation had been suppressed entirely by the ionotophoretic application of GABA. In this case, it may be argued that the reduced firing seen after the adaptation period could be due to a certain amount of GABA lingering in the vicinity of the cell. This possibility is excluded by the fact that after application of the same current of GABA over a similar duration, but without the presentation of the adapting pattern, no adaptation effects were seen (Fig. 1).

In Table 1 the mean of the above results can be seen for all the cells tested. Despite complete suppression of the cells' responses during adaptation by the iontophoretically applied GABA, adaptation occurred to the same extent as in controls (conditions $A$ and B). Prolonged application of GABA for an equivalent period without presentation of the highcontrast adapting gratings produced no comparable adaptation (condition $\mathrm{C}$ ). Since the iontophoretic GABA cannot be expected to suppress the occurrence of excitatory postsynaptic potentials (EPSPs) all over the postsynaptic surface, the excitatory amino acid, L-glutamic acid was iontophoretically applied without the presentation of the grating, to see whether the excitation of the cell per se had the effect of reducing the sensitivity of the cell. The glutamate current was adjusted to yield spike activity equal to or greater than that produced by the adapting pattern, but below that which produced depolarization block. Such a procedure did not lead to any reduction of responses to a subsequently presented test grating (condition $\mathrm{D}$ ).

In some neurons, the effect of iontophoretic application of bicuculline on adaptation was tested. The dose of bicuculline methiodide was adjusted to block the effect of iontophoretically applied GABA, which would otherwise completely suppress the response of the cell to an optimum stimulus (see Experimental Procedures). Adaptation was then again measured during the administration of bicuculline. Figure 2 shows that cells exhibited adaptation during bicuculline application as well. The magnitude of this adaptation was comparable with the control situation, as can be seen from the means in Table 1 (conditions $\mathrm{A}$ and $\mathrm{E}$ ).

\section{DISCUSSION}

Pattern adaptation was not affected by suppressing the cell activity by the application of the inhibitory transmitter, GABA in the close vicinity of the cell. That spike activity is not necessary for adaptation to occur is consistent with earlier reports that cortical neurons can be adapted by stimuli which do not themselves evoke responses. ${ }^{17,21}$ However, it may be argued that occurrence of EPSPs may be sufficient to lead to adaptation even if action potentials are suppressed. But the absence of any reduction in cell sensitivity after the application of glutamate excludes the possibility of neuronal fatigue from excitatory activity being the basis of adaptation.

It has been postulated that a cell stimulated by the presentation of a high-contrasi optimal grating concomitantly experiences considerable inhibition and this effect persists leading to the lowered sensitivity 

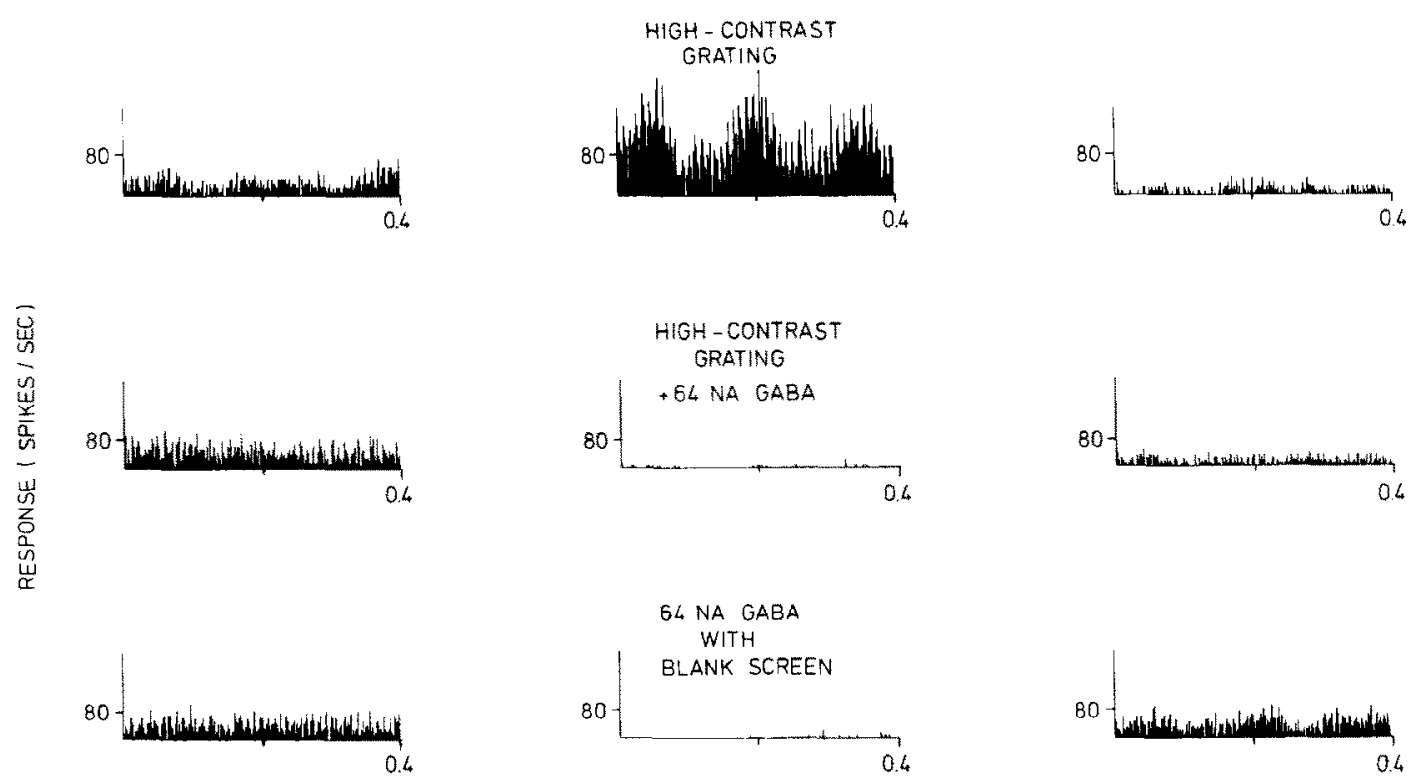

TIME $(S E C)$

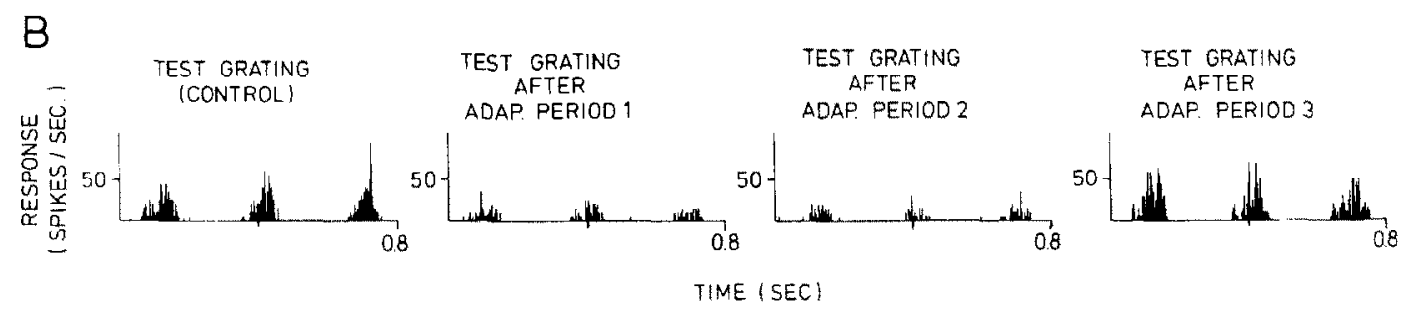

Fig. 1. Peristimulus time histograms for a complex (A) and a simple (B) cell after various adaptation procedures using moving sin wave grating stimuli. (A) Each horizontal row shows peristimulus time histograms from an interleaved set of recording conditions, with the control response to the low-contrast $(5 \%)$ grating on the left, the response during the adaptation to a high-contrast $(40 \%)$ in the middle (except in the bottom row) and the response to the low-contrast grating presented immediately after the adaptation period on the right. The stimulus was a 0.16 cycles/deg grating moving at $6.84 \mathrm{~Hz}$. Each stimulus condition was presented for $12.8 \mathrm{~s}$ and the whole cycle repeated four times, with the control test grating always preceded by a period of $15 \mathrm{~s}$, during which no grating was presented on the screen to allow recovery from adaptation. The middle row represents the situation where $64 \mathrm{nA}$ of GABA was microiontophoretically applied during the presentation of the high-contrast adaptation grating. The GABA could completely suppress the response of the cell. The few spikes seen were during the first sweep $(400 \mathrm{~ms})$ when GABA had not had its full effect. The bottom row represents the situation where the same current of GABA was applied over the same period as above, but no grating was presented on the screen, which was maintained at its mean luminance. (B) Peristimulus time histograms from a similar adaptation protocol as above are shown for presentation of the control test grating and the test grating after the three adaptation periods (numbered 1,2 and 3, corresponding to the top, middle and bottom rows in $\mathrm{A}$ ). The stimulus was a 0.64 cycles/deg grating moving at $3.42 \mathrm{~Hz}$. The test contrast used was $40 \%$, since adaptation effects could be clearly seen even using this high contrast.

seen subsequently. ${ }^{8}$ But the inhibited state of the cell itself cannot be the basis of adaptation, since application of GABA, without the presentation of the adapling patterns did not reduce the sensitivity of the cell. Thus adaptation of an individual cortical cell is independent of the excitatory and inhibitory activity of that particular neuron, and possibly reflects a change in the input to the cell. Such a change is unlikely to be in the geniculate input, since neurons in the dLGN show no adaptation effects. ${ }^{17,20,21}$ Further, interocular transfer of adaptation in area 17 neurons, ${ }^{16}$ the spatial frequency and orientation specificity of the effect ${ }^{17,19}$ and recent studies of motion after-effect in striate neurons ${ }^{12,13}$ suggest that 
Table 1. Response amplitudes $\left(R_{2}\right)$ to test gratings at different adaptation conditions (A E), relativ to the control response to the test grating prior to adaptation $\left(R_{1}\right)$

\begin{tabular}{lccccc}
\hline & $\mathrm{A}$ & $\mathrm{B}$ & $\mathrm{C}$ & $\mathrm{D}$ & $\mathrm{E}$ \\
\hline Relative & & & & & \\
response & $0.55 \pm 0.032$ & $0.52 \pm 0.043$ & $0.89 \pm 0.055$ & $1.11 \pm 0.084$ & $(n=25)$ \\
$\left(R_{2} / R_{1}\right)$ & $(n=30)$ & $(n=20)$ & $(n=4)$ & $\begin{array}{c}0.62 \pm 0.052 \\
(n=8)\end{array}$ \\
\hline
\end{tabular}

If the response is totally unaffected by the adaptation procedure, the ratio would be 1 . The mean ratio is shown for each adaptation condition, along with the standard error. For each cell, the contrast of the test grating and the contrast of the adapting grating were so chosen that the adaptation reduced the cell's response by nearly half (represented by condition A, corresponding to adaptation period 1 in Fig. 1). Condition B: response to test grating after adaptation period 2, during which grating of the same contrast and duration as in $\mathrm{A}$ was presented along with iontophoresis of GABA that was sufficient to suppress all responses. Condition $\mathrm{C}$ : response to test grating after adaptation period 3, during which the same current of GABA as in B was ejected over the same period, but without presentation of the grating. Condition $D$ : responso to test grating after an adaptation period, during which glutamate was ejected over the same period as in $\mathrm{A}-\mathrm{C}$, but without the presentation of the grating, and at an ejection current that produced a mean spike count comparable with that during adaptation period 1 . Condition $\mathrm{E}$ : response to test grating after an adaptation period similar to that in A, during which bicuculline methiodide was applied at an ejection current that could block the effect of GABA which was iontophoresed at a current at least equal to that used in condition B. Bicuculline ejection was continued during all phases of the cycle, both adapting and non-adapting. Controls were also done in a few cells to exclude current effects, by ejecting $\mathrm{Na}^{+}$ions from a barrel containing $3 \mathrm{M} \mathrm{NaCl}$. This procedure had no effect on adaptation.

pattern adaptation occurs at the cortical level. The putative intracortical input that modulates the activity during adaptation cannot be inhibitory since adaptation occurred to the normal extent despite iontophoretic application of bicuculline. This is consistent with the shift in contrast-response functions of cortical cells reported during adaptation in the presence of local application of bicuculline." An

\section{A}

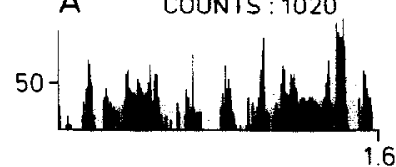

482
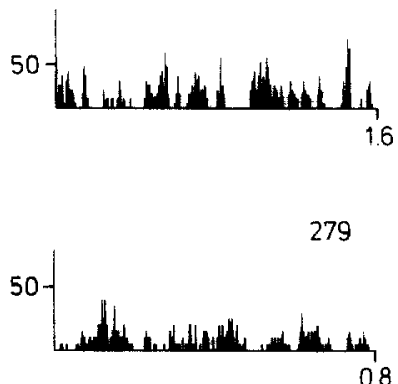

274

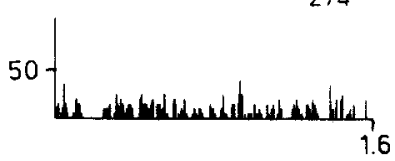

764



8

0.8

TIME (SEC)

Fig. 2. Responses of three complex $(A-C)$ and one simple (D) cell to test gratings during the application of bicuculline methiodide (condition E of Table 1). Peristimulus time histograms on the left are control ones done without prior adaptation to a high-contrast grating, and those on the right were done immediately after exposure to the adapting grating. Optimum spatial $(0.16$ cycles $/ \operatorname{deg}$ for $\mathrm{A}$ and $\mathrm{B}$, and 0.48 and 0.32 cycles $/ \mathrm{deg}$ for $C$ and $D$, respectively) and temporal $(1.71 \mathrm{cycles} / \mathrm{s}$ in $A$ and $C$ and $3.42 \mathrm{cycles} / \mathrm{s}$ in $B$ and D) frequencies were used. The contrasts for the adapting and test gratings were chosen as explained earlier. 
alternative interpretation of the results would be that adaptation is mediated by bicuculline insensitive GABA $_{b}$ receptors $^{6}$ or through other inhibitory transmitters. But iontophoretic studies using the $\mathrm{GABA}_{b}$ antagonist, phaclofen have shown that the inhibition associated with these receptors lack orientation and direction selectivities. ${ }^{2}$ There is also at present no evidence for any functional non-GABA inhibition in the visual cortex, let alone the spatial frequency and orientation specificities that would be required of this inhibition to explain pattern adaptation

\section{CONCLUSION}

The adaptation seen in a striate neuron reflects a change in the intracortical excitatory inputs to that cell. Cross-correlation studies in area $17^{22}$ suggest that long-range horizontal connections are largely between cell clusters of similar orientation preference and are excitatory in nature. There is also morphological evidence that horizontal projections in the visual cortex are excitatory. 70,14 Adaptation could thus be the expression of reduced activity in a stimulus specific co-operative network whose effect on each individual cell is largely mediated through excitatory connections. The excitatory network may have a facilitatory or synchronizing role during normal perception, ${ }^{11.18}$ and adaptation can be looked upon as a reduction of these lateral excitatory interactions. The functional role of such a reduction may be to prevent excessive activity in the facilitatory network leading to plastic changes like long-term potentiation in adult primary sensory areas. which need to be maintained as reliable processors of incoming sensory information.

\section{REFERENCES}

1. Albrecht D. G., Farrer S. B. and Hamilton D. B. (1984) Spatial contrast adaptation characteristics of neurones recorded in the cat's visual cortex. J. Physiol. 347, 713-739.

2. Baumfalk U. and Albus K. (1988) Phaclofen antagonizes baclofen-induced suppression of visually evoked responses in the cat's striate cortex. Brain Res. $\mathbf{4 6 3}, 398.402$

3. Blakemore $C$ and Campbell F. W. (1969) On the existence in the human visual system of neurones selectively sensitive to the orientation and size of retinal images. $J$. Physiol. 203, 237-260.

4. Blakemore C., Carpenter R. H. S. and Georgeson M. A. (1970) Lateral inhibition between orientation detectors in the human visual system. Nature 228, 37-39.

5. Blakemore C. and Sutton P. (1969) Size adaptation: a new aftereffect. Science 166, 245-247.

6. Bowery N. G., Price G. W., Hudson A. L., Hill D. R., Wilkin P. and Turnbull M. J. (1984) GABA receptor multiplicity. Visualization of different receptor types in the mammalian CNS. Neuropharmacology 23, 219 -231.

7. Creutzfeldt O. D., Garey L. J., Kuroda R. and Wolf J. R. (1977) The distribution of degenerating axons after small lesions in the intact and isolated visual cortex of the cat. Expl Brain Res. 27, $419-440$.

8. Dealy R. S. and Tolhurst D. J. (1974) Is spatial adaptation an after-effect of prolonged inhibition? J. Physiot. 241, 261- 270 .

9. DeBruyn E. J. and Bonds A. B. (1986) Contrast adaptation in cat visual cortex is not mediated by GABA. Brain Res. $383,339 \quad 342$

10. Fisken R. A., Garey L. J. and Powell T. P. S. (1975) The intrinsic association and commisural connections of area 17 of the visual cortex. Phil. Trans. R. Soc. 272, 487-536.

11. Gray C. M., Koenig P. Engel A. K. and Singer W. (1989) Oscillatory responses in cat visual cortex exhibit inter-columnar synchronization which reflects global stimulus properties. Nature $\mathbf{3 3 8}, 334-337$.

12. Hammond P. and Mouat G. S. V. (1988) Neural correlates of motion after-effects in cat striate cortical neurones: monocular adaptation. Expl Brain Res. 72, 1-20.

13. Hammond P. and Mouat G. S. V. (1988) Neural correlates of motion after-effects in cat striate cortical neurones: interocular transfer. Expl Brain Res. 72, 21-28.

14. LeVay S. (1988) Patchy intrinsic projections in visual cortex, area 18, of the cat: morphological and immunocytochemical evidence for an excitatory function. J. comp. Neurol. 269, 265-274.

15. Maddess T., McCourt M. E., Blakeslee G. and Cunningham R. B. (1988) Factors governing the adaptation of cells in area 17 of the cat visual cortex. Biol. Cybern. 59, 229-236.

16. Maffei L., Berardi $N$. and Bisti $S$. (1986) Interocular transfer of adaptation aftereffect in neurons of areas 17 and 18 of split chiasm cats. J. Neurophysiol. 55, 966-976.

17. Maffei L. and Fiorentini A. (1973) Neural correlate of perceptual adaptation to gratings. Science 182, 1036-1038.

18. Mitchison $G$. and Crick F. (1982) Long axons within the striate cortex their distribution, orientation and patterns of connection. Proc. natn. Acad. Sci. U.S.A. 79, 3661-3665.

19. Movshon J. A. and Lennie P. (1979) Pattern-selective adaptation in visual cortical neurones. Nature 278,850 - 852.

20. Ohzawa I. Sclar G, and Freeman R. D. (1982) Contrast gain control in the cat visual cortex. Nature 298, 266 268.

21. Ohzawa I., Sclar G. and Freeman R. D. (1985) Contrast gain control in the cat visual system. $J$. Nourophywiol. 54, $651 \cdot 667$.

22. Ts*o D. Y., Gilbert C. D. and Wiesel T. N. (1986) Relationships between horizontal interactions and functional architecture in cat striate cortex as revealed by cross-contelation analysis. $J$. Neurosci. 6, $1160-1170$.

23. Vidyasager T. R. (1984) Contribution of inhibitory mechanisms to the orientation sensitivity of cat dLGN neurones. Expl Brain Res. 55, $192-195$. 